\title{
L'azote non protéique du lait : facteurs de variation
}

\author{
par \\ M. JOURNET*, R. VERITE* \\ Laboratoire de la Production laitière C.R.Z.V. de Theix \\ 63110 Beaumont \\ et B. VIGNON** \\ E.N.S.A. - 54000 Nancy
}

La fraction azotée non protéique du lait a une valeur nutritionnelle presque nulle aussi bien pour les humains que pour les jeunes ruminants si l'on excepte le peu d'acides aminés libres qu'elle renferme (15 p. 100 en moyenne de l'azote non protéique). Elle n'intéresse pas non plus le fromager puisqu'elle n'est pas coagulable*. S'il importe de connaître ses variations, c'est principalement parce que le dosage d'azote total par la méthode Kjeldahl peut donner une idée inexacte de la valeur protéique du lait puisqu'il mesure en fait la somme de l'azote protéique et de l'azote non protéique.

\section{I. - LES FRACTIONS NON PROTEIQUES DU LAIT ET LEUR ORIGINE}

\section{1) Teneurs moyennes et variations}

L'azote non protéique (ANP) comprend principalement de l'urée, mais en proportion très variable : de 20 à 75 p. 100. La teneur en azote uréique peut en effet varier de 7 à $15 \mathrm{mg}$ par $100 \mathrm{~g}$ de lait de vache dans des conditions assez normales et de 5 à $30 \mathrm{mg}$ dans des conditions extrêmes (Shahani et Sommer, 1951 ; Ide et al., 1966 ; Mc Dowell, 1972 ; Vignon et Laurent, 1972). Les autres composants de l'ANP sont les acides aminés (10 à 20 p. 100 de l'ANP), la

* Elle est d'ailleurs ultra-filtrable (Maubois et al., 1971) si bien qu'elle ne peut pas non plus être incorporée dans le fromage préparé à partir de lait ultrafiltré. 
TABLEAU 1. - Fractions non protéiques du lait. Echantillons de laits de troupeaux ou de lots d'animaux

\begin{tabular}{|c|c|c|c|c|c|c|}
\hline \multirow[b]{2}{*}{ Auteurs } & \multirow{2}{*}{$\begin{array}{c}\text { Nombre } \\
\text { d'échantillons }\end{array}$} & \multicolumn{3}{|c|}{$\mathrm{mg} \mathrm{d}^{\prime} \mathrm{N} / 100 \mathrm{~g}$ de lait } & \multirow{2}{*}{$\frac{\mathrm{ANP}}{\mathrm{AT}} \times 100$} & \multirow{2}{*}{$\frac{\text { N urée }}{\text { ANP }} \times 100$} \\
\hline & & ANP & urée & $\begin{array}{l}\text { ANP } \\
\text { autre que } \\
\text { l'urée }\end{array}$ & & \\
\hline \multirow[t]{6}{*}{$\begin{array}{l}\text { Vignon, Brun-Bellut, } 1972 \\
\text { (non publié) }\end{array}$} & $\begin{array}{l}\text { mai - juin } 1972 \\
\text { (3 échantillons/vache) }\end{array}$ & & & & & \\
\hline & troupeau I 70 vaches & $25,0 \pm 2,5$ & $11,8 \pm 1,9$ & 13,2 & 4,7 & 47 \\
\hline & troupeau II 49 vaches & $28,2 \pm 2,7$ & $15,9 \pm 2,6$ & 12,2 & 5,4 & 57 \\
\hline & troupeau III 35 vaches & $32,1 \pm 2,6$ & $18,9 \pm 2,5$ & 13,6 & 6,1 & 58 \\
\hline & troupeau IV 63 vaches & $25,6 \pm 3,2$ & $13,2 \pm 2,9$ & 12,3 & 4,7 & 52 \\
\hline & troupeau V 59 vaches & $32,9 \pm 3,3$ & $20,0 \pm 2,8$ & 13,9 & 6,1 & 61 \\
\hline Shahani, Sommer, 1951 & 6 & $25-32$ & $6-20$ & $12-19$ & & $24-63$ \\
\hline Mc Dowell, 1972 & $\begin{array}{l}433 \text { (Frisonnes) } \\
159 \text { (Jersayaises) }\end{array}$ & $\begin{array}{l}24-54 \\
22-51\end{array}$ & $\begin{array}{r}12-29 \\
9-31\end{array}$ & & $\begin{array}{l}4,9-10,2 \\
3,7-8,7\end{array}$ & $\begin{array}{l}41-67 \\
33-79\end{array}$ \\
\hline Waite et al., 1968 & 3 par essai : Essai I & $\begin{array}{l}25-26 \\
22-23\end{array}$ & $\begin{array}{c}9-11 \\
9\end{array}$ & $\begin{array}{l}14-17 \\
12-14\end{array}$ & $\begin{array}{l}5,0-5,1 \\
4,4-4,6\end{array}$ & $\begin{array}{l}34-43 \\
39-43\end{array}$ \\
\hline Ide et al., 1966 & $\begin{array}{l}7 \text { rations de nature très } \\
\text { différente }\end{array}$ & $18-40$ & $4-30$ & $11-18$ & & $29-74$ \\
\hline
\end{tabular}


créatine ( 3 à 6 p. 100), l'acide urique (2 à 4 p. 100), l'ammoniaque (1 à $5 \mathrm{p} .100$ ) et la créatinine (environ $1 \mathrm{p} .100$ ), etc. La teneur du lait en ces composants (autres que l'urée) varie assez peu (tab. 1): en moyenne 12 à $15 \mathrm{mg}$ d'N par $100 \mathrm{~g}$ de lait (11 à $18 \mathrm{mg}$ à l'extrême : Shahani et Sommer, 1951 ; Venkatappaiah et Basu, 1952). Les variations de la teneur en ANP du lait sont dues essentiellement à celles de l'urée.

L'azote non protéique ne représente qu'une faible fraction de l'azote du lait, entre 5 et 6 p. 100, dans les résultats d'enquêtes effectuées sur des laits de mélange de grande région (Reinart et Nesbitt, 1956 ; Burton, 1967 ; Nickerson, 1960). Des valeurs supérieures à 6 ont cependant été observées : 6,16 sur des laits de l'Ontario provenant pour 80 p. 100 de troupeaux de vaches Holstein (Szijarto et al., 1973) et 6,46 sur des troupeaux frisons de NouvelleZélande alimentés presque exclusivement au pâturage (Mc Dowell, 1972). Ainsi, la teneur en ANP est le plus souvent comprise entre 25 et $30 \mathrm{mg}$ d'azote par $100 \mathrm{~g}$ de lait $4 \mathrm{p} .100$, soit l'équivalent de 1,6 à $1,9 \mathrm{~g}$ de matière azotée totale $(\mathrm{N} \times 6,39)$ par $\mathrm{kg}$ de lait.

\section{2) Variations comparées des fractions azotées protéique et non protéique du lait}

Les variations de teneur en azote de la fraction non protéique du lait sont faibles par rapport aux variations de teneur en azote total ou de teneur en azote protéique du lait. C'est ce que montrent (tab. 2) les enquêtes effectuées sur des laits de grand mélange (troupeau ou usine) issus de zones géographiques très diverses (Californie, Nouvelle-Zélande) avec des conditions très variables de milieu, de race et de conduite d'élevage. Généralement, les variations de teneur en azote total sont 6 à 15 fois plus importantes que les variations de teneur en ANP. La teneur en lactalbumine + lactoglobuline + protéose peptone est moins variable que la teneur en caséine mais cependant 2 à 3 fois plus que celle en ANP (Nickerson, 1960). Dans des conditions de milieu très homogènes (saison donnée, aliments et rationnements identiques, etc.) la variabilité entre troupeaux du taux d'azote protéique est faible et se rapproche plus alors de celle du taux d'ANP. C'est ce qu'ont observé Vignon et Brun-Bellut (1972) sur des troupeaux d'une même zone géographique se trouvant tous au pâturage à une même date.

\section{3) Origine des fractions non protéiques}

L'ANP qui est constitué pour une part importante par de l'urée provient des matières azotées alimentaires d'origine protéique et non protéique. On sait en effet que la totalité de l'azote non protéique et 40 à 70 p. 100 de l'azote protéique sont transformés en ammoniaque dans le rumen. Une partie de l'ammoniaque est réutilisée et transformée en protéines microbiennes, mais l'excès traverse l'épithé- 
Variabilités comparées des teneurs en azote total, en azote caséine et en azote non protéique du lait, sous l'influence de différents facteurs : troupeaux, régions, individus, saisons

\begin{tabular}{|c|c|c|c|c|c|}
\hline \multirow{2}{*}{ Auteurs } & \multirow{2}{*}{ Facteurs de variation } & \multicolumn{4}{|c|}{$\begin{array}{l}\text { Ecart entre les valeurs extrêmes } \\
\left(\mathrm{mg} \mathrm{d}^{\prime} \mathrm{N} / 100 \mathrm{~g} \text { de lait }\right)\end{array}$} \\
\hline & & $\begin{array}{l}\mathrm{N} \text { total } \\
\text { (1) }\end{array}$ & $\begin{array}{l}\mathrm{N} \text { caséine } \\
(2)\end{array}$ & $\begin{array}{c}\text { ANP } \\
(3)\end{array}$ & $(1) /(3)$ \\
\hline $\begin{array}{l}\text { Nickerson (1960) } \\
\text { Californie (U.S.A.) }\end{array}$ & 6 régions & 77 & 63 & 3 & 25 \\
\hline $\begin{array}{l}\text { Hartland (1955) } \\
\text { Ensemble des U.S.A. }\end{array}$ & 81 laits de troupeaux & 288 & 253 & 19 & 15 \\
\hline Mc Dowell (1972) & 433 laits de troupeaux frisons & 169 & & 30 & 6 \\
\hline Nouvelle-Zélande & 159 laits de troupeaux jersey & 251 & & 29 & 9 \\
\hline $\begin{array}{l}\text { Vignon (1972) } \\
\text { Est de la France }\end{array}$ & 4 troupeaux (mai-juin) & 20 & & 8 & 3 \\
\hline $\begin{array}{l}\text { Nickerson (1960) } \\
\text { Californie (U.S.A.) }\end{array}$ & 4 saisons & 33 & 27 & 0 & \\
\hline $\begin{array}{l}\text { Burton (1967) } \\
\text { Sud Angleterre }\end{array}$ & 12 mois $\begin{array}{l}\text { troupeau I } \\
\text { troupeau II }\end{array}$ & $\begin{array}{l}73 \\
88\end{array}$ & $\begin{array}{l}54 \\
78\end{array}$ & $\begin{array}{r}13 \\
9\end{array}$ & $\begin{array}{r}6 \\
10\end{array}$ \\
\hline $\begin{array}{l}\text { Shahani (1951) } \\
\text { Wisconsin (U.S.A.) }\end{array}$ & 14 laits de mélange & 91 & 77 & 11 & 8 \\
\hline Shahani (1951) & 14 individus & 107 & 74 & 8 & 13 \\
\hline
\end{tabular}


lium du rumen et est transformé en urée par le foie ; l'urée provient également pour une plus faible part des acides aminés en excès issus de la digestion enzymatique dans l'intestin des protéines non dégradées dans le rumen. Les cellules épithéliales de la glande mammaire sont très perméables à l'urée, de sorte qu'on observe une liaison très étroite entre la teneur en urée $(\mathrm{mg} / 100 \mathrm{ml})$ du plasma sanguin $(x)$ et celle du lait $(y)(r=0,82 ; y=0,91 x+0,4)$. Cette relation a été obtenue à partir de 80 mesures sur 30 vaches (Vérité). Peskett (1934) et Wheelock et Rook (1966) et Ide et al. (1966) ont également observé des teneurs en urée sensiblement identiques dans le lait et dans le sang.

On comprend pourquoi les teneurs élevées en ANP sont dues principalement à un excès d'apport azoté. Inversement, un apport important d'énergie sous forme de glucides facilement digestibles, de l'amidon par exemple, en accroissant les synthèses protéiques dans le rumen à partir de l'ammoniaque (et dans l'organisme) fait diminuer le taux d'urée sanguine et donc le taux d'urée du lait.

On comprend aussi pourquoi ces variations du taux d'ANP ne sont que passagères puisqu'elles sont simplement le reflet d'un déséquilibre de la ration et qu'elles sont plus faibles que celles du taux d'azote protéique qui dépend de l'animal (race, individu...), de l'état physiologique (hormones) et de l'état nutritionnel (niveau des apports énergétiques et disponibilité en acides aminés).

\section{II. - INFLUENCE DES FACTEURS DU MILIEU : ALIMENTATION ET SAISON}

\section{1) Niveau d'apport azoté}

La teneur en ANP du lait dépend avant tout du niveau des apports azotés de la ration (revue de Rook, 1961). Rook et Line (1962) ont montré que lorsque les apports azotés passaient de 70 à 165 p. 100 des valeurs recommandées, le taux d'ANP du lait passait de 16 à $33 \mathrm{mg} \mathrm{N}$ par $100 \mathrm{~g}$. En fait ce serait essentiellement la fraction urée du lait qui augmenterait puisque Ide et al. (1966) ont observé que le taux d'urée du lait passait de 7 à $21 \mathrm{mg}$ par $100 \mathrm{~g}$ quand les apports azotés passaient de 70 à 170 p. 100 des recommandations. Avec différents types de rations hivernales (fig. 1, Vérité et Journet, résultats non publiés) l'accroissement du taux azoté des rations (de 12 à 18 MAT p. 100 MS) correspond à un accroissement sensiblement linéaire du taux d'urée plasmatique (de 7,5 à $18,5 \mathrm{mg}$ $\mathrm{N} / 100 \mathrm{~g}$ ) et donc aussi du taux d'urée dans le lait (puisque les teneurs en urée du lait et du sang sont très voisines). On peut donc estimer qu'en moyenne le taux d'urée du lait augmente de $15 \mathrm{mg}$ $\mathrm{N} / 100 \mathrm{~g}$ (soit l'équivalent de $1 \mathrm{~g}$ de MAT par $\mathrm{kg}$ de lait) quand la 
teneur en matières azotées de la ration est multipliée par 2. Cette variation est relativement modeste comparée à l'ampleur de la variation de l'apport azoté.

L'augmentation du taux d'ANP du lait avec l'apport azoté est d'autant plus importante que l'apport d'énergie est plus faible (Orth et Kaufmann, 1964). Ainsi dans l'essai de Ide et al. (1966), le taux d'urée dans le lait le plus élevé ( $30 \mathrm{mg} \mathrm{N} / 100 \mathrm{~g}$ ) a correspondu à des apports azotés élevés ( 2 fois les recommandations) et à une sousalimentation énergétique importante ( 50 p. 100 des recommandations). A l'opposé, le taux le plus faible (3,7 mg N par $100 \mathrm{~g}$ ) a été obtenu avec des apports azotés faibles et un excès d'énergie (120 p. 100 des recommandations). De même, l'augmentation du rapport énergie/azote de la ration de 1 à 1,5 a fait diminuer le taux d'ANP du lait de $9 \mathrm{mg}$ par $100 \mathrm{~g}$ (Gordon et Forbes, 1970).

\section{2) Nature du régime alimentaire}

Les variations du taux d'ANP du lait selon le type de ration peuvent en grande partie s'expliquer par les variations du niveau des apports azotés (et énergétiques), comme nous l'avons observé (fig. 1) avec différentes rations hivernales à base d'ensilage d'herbe ( + pulpes ou betteraves) et d'ensilage de maïs (avec ou sans foin et urée). A taux azoté égal des rations, des différences dans la solubilité des matières azotées alimentaires peuvent dans certains cas entraîner des différences, relativement faibles, du taux d'ANP du lait. Ainsi Renner et Gonc (1972) ont observé un accroissement de 3 à $6 \mathrm{mg} \mathrm{N} / 100 \mathrm{~g}$ avec des rations d'ensilage comparées à une ration à base de foin.

La mise à l'herbe entraîne des modifications très différentes selon les auteurs. Dans certains cas, on observe des baisses de taux d'ANP du lait (Jarrige, 1953) et dans d'autres cas des hausses (Devuyst et al., 1964 ; Vignon et Laurent, 1972) selon que le taux azoté des rations distribuées avant la mise à l'herbe est plus ou moins élevé que celui de l'herbe. L'augmentation du taux d'ANP du lait au pâturage peut être importante. Ainsi en 1969-70-71, ce taux a été supérieur de 30 à 50 p. 100 pour les animaux mis à l'herbe comparés à des animaux maintenus à l'étable. Cet accroissement est fugace puisque $20 \mathrm{j}$ après la mise à l'herbe il n'y a plus de différence (Vignon et Laurent, 1972). Dans d'autres essais (Vérité et Journet, non publiés), nous avons observé, au printemps, 1 mois après la mise à l'herbe, que le taux d'urée plasmatique variait de 7 à $18 \mathrm{mg} \mathrm{N}$ selon les années, parce que l'herbe se trouvait à des âges physiologiques très différents. Devuyst et al. (1964) ont également observé des variations relativement importantes (10 $\mathrm{mg} \mathrm{N} / 100 \mathrm{~g})$ au cours d'une saison de pâturage. Les valeurs maximum sont généralement observées à la mise à l'herbe au printemps et sur des pâturages d'automne riches en azote mais pauvres en glucides solubles 
(Szijardo et al., 1973). L'ANP peut alors représenter jusqu'à 7,5 p. 100 de l'azote total du lait.

\section{3) Apport d'azote non protéique dans la ration}

Les essais réalisés pour connaître l'effet d'un apport d'urée dans la ration sur le taux d'urée (ou d'ANP) dans le lait ont donné des résultats très variables sans doute à cause de la grande diversité des conditions de réalisation (en particulier en ce qui concerne le niveau des apports azotés). Le remplacement de l'azote des tourteaux (ou d'une partie de l'azote des tourteaux) par de l'azote provenant de l'urée ne modifie généralement pas (ou peu) le taux d'urée et d'ANP du lait, sauf si l'urée est employée dans des conditions inadéquates (taux d'azote excessif de la ration ou rations riches en azote soluble). C'est ce que montrent les résultats relatifs aux teneurs

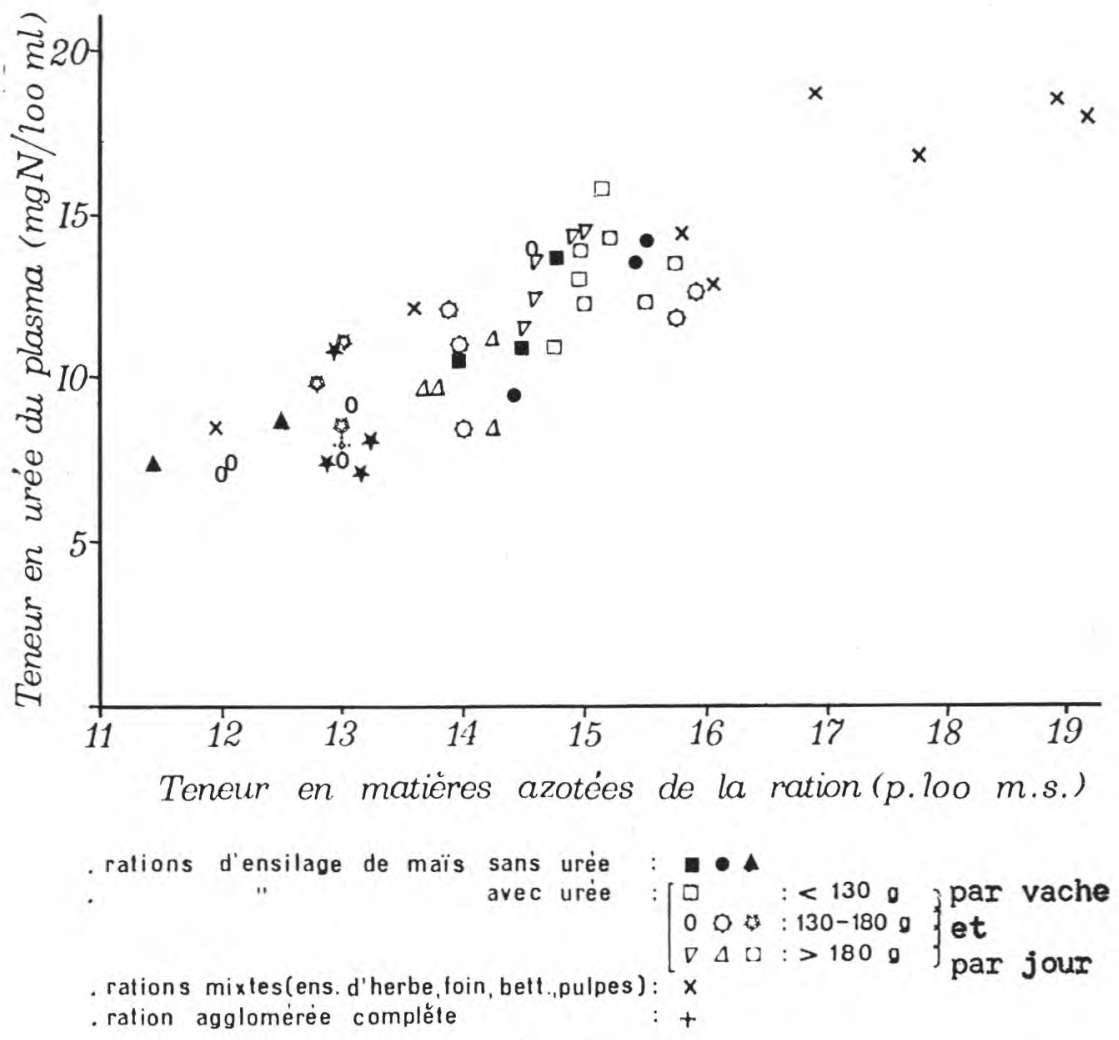

fig. 1

Influence de la nature des rations et de leur teneur en matières azotées sur la teneur en urée du plasma sanguin 
en urée du sang obtenus au Laboratoire de la Production laitière avec des rations d'ensilage de maïs où l'apport d'urée a varié selon les essais de 100 à $220 \mathrm{~g}$ par vache et par jour (fig. 1). Dans les nombreux essais effectués par Virtanen (1967), avec des rations synthétiques et très énergétiques où tout l'apport azoté de la ration était sous forme non protéique, le taux d'ANP du lait a été normal (4,2 p. 100 de l'azote total) et même inférieur à celui observé avec des rations classiques utilisant des sources d'azote protéique. Ces résultats sont confirmés par d'autres auteurs (Iwata, 1958 ; Waite et al., 1968 ; Robertson et Dixon, 1969). Dans d'autres cas, l'apport d'urée a occasionné un accroissement de 8 à $10 \mathrm{mg}$ d'azote non protéique/100 g de lait (Vignon, 1972 ; Senft et Klobasa, 1969). Pour le premier auteur la teneur en matières azotées totales de la ration était légèrement plus élevée avec l'urée qu'avec le tourteau ; pour le deuxième auteur on ne connaît pas le taux azoté des rations.

\section{4) La saison}

La saison a généralement peu d'effet sur la teneur en ANP du lait. C'est ce qui ressort de l'étude de Nickerson (1960) en Californie qui a porté sur 258 laits de mélange provenant de 6 régions, l'année ayant été divisée en 4 saisons. Mc Dowell (1972) n'a pas non plus observé de variations mensuelles ou a observé des variations faibles de 5 à $10 \mathrm{mg}$ d'ANP/100 g de lait sur des troupeaux de vaches Frisonnes et Jersiaises. Burton (1967) en Angleterre a cependant observé des variations plus importantes atteignant jusqu'à 14 mg/100 g de lait dans un troupeau de vaches Frisonnes. De même Grappin (1973) a constaté pendant 2 années, dans le Jura, que la proportion d'ANP dans l'azote du lait passait de 4,5 p. 100 durant les mois d'hiver à 5,5 p. 100 durant les mois d'été. Les mêmes variations ayant été observées par Szijarto et al. (1973) au Canada. Il est probable que les variations observées par ces auteurs sont d'origine alimentaire, les valeurs les plus élevées correspondent à la période de pâturage et proviennent probablement d'herbe assez riche en azote. Ces variations avec la saison du taux d'azote non protéique ont été de 8 à 10 fois moins grandes que celles du taux d'azote total ou d'azote protéique (Mc Dowell, 1972).

\section{III. - FACTEURS LIES A L'ANIMAL}

La teneur en ANP du lait semble très peu influencée par le stade physiologique ou les caractéristiques individuelles des vaches. Elle ne varie pas avec le stade de lactation, exception faite de la $1^{\text {re }}$ semaine où elle passe de plus de $50 \mathrm{mg}$ d'azote $/ 100 \mathrm{~g}$ de lait le $1^{\text {er }}$ jour, à $30 \mathrm{mg}$ le $8^{\mathrm{e}}$ jour (Vignon, 1973). Dans l'étude de Rook et Campling (1965), elle diminue encore de 4 à $5 \mathrm{mg}$ au cours des 
5 semaines suivantes. Elle semble diminuer avec l'âge d'environ $5 \mathrm{mg}$ entre la $1^{\text {re }}$ et la $5^{\mathrm{e}}$ lactation (Klobasa, 1969). Peu d'auteurs ont étudié les variations individuelles du taux d'ANP. L'écart le plus important observé entre 14 vaches par Shahani et Sommer (1951) a été de $8 \mathrm{mg}$ d'ANP/100 g de lait. Vignon et Brunbellut (1972) dans une étude portant sur 4 troupeaux de 50 à 70 vaches chacun ont observé un écart-type entre animaux de 2,5 à $3,3 \mathrm{mg}$ d'ANP/100 $\mathrm{g}$ (coefficient de variation de 10 p. 100). Sur des troupeaux de 50 à 80 vaches, Grappin (1973) a constaté que le pourcentage d'azote non protéique dans le lait pouvait varier dans la proportion de 1 à 2 . Wheelock et al. (1966) ont montré que le taux d'ANP ne variait pas au cours de la traite mais qu'il pouvait être accru en cas d'accident de la mamelle. Il est plus élevé à la traite du matin qu'à la traite du soir (Klobasa, 1969).

\section{CONCLUSION}

En conclusion, l'azote non protéique constitue une fraction mineure de l'azote du lait et ses variations, sous l'influence des facteurs du milieu et des facteurs liés à l'animal, sont faibles comparées à celles de l'azote protéique. Ceci pourrait justifier dans une certaine mesure que, lors du dosage de l'azote total, on ne se préoccupe pas de l'azote non protéique. Les variations du taux d'azote total entre troupeaux en fonction du régime alimentaire et de la saison sont, en effet, dues essentiellement à l'azote protéique et plus particulièrement à l'azote de la caséine.

Cependant, le paiement du lait à la matière azotée justifiait qu'on précise les risques d'un accroissement de la teneur en azote non protéique dans certaines conditions. On s'aperçoit qu'une augmentation notable de ce taux pouvant atteindre l'équivalent de $1 \mathrm{~g}$ de matières azotées $(\mathrm{N} \times 6,25)$ par $\mathrm{kg}$ de lait n'est obtenue qu'avec de forts excédents d'apports azotés dans des rations par ailleurs déficitaires en énergie. C'est sur des pâturages d'herbe très jeune, fortement fertilisés et principalement à l'automne que ceci a le plus de chances de se trouver réalisé. Mais l'urée utilisée avec des rations d'ensilage de maïs en remplacement (et non en supplément) d'une partie des tourteaux n'accroît pas ou accrôit peu le taux d'azote non protéique du lait.

Les méthodes de dosage rapides de l'azote et notamment la méthode au Noir Amido sont des méthodes spécifiques de dosage des protéines. Toutefois, selon la méthode de référence (Kjeldahl) qui est employée pour étalonner les appareils : $\mathrm{N}$ total $\times 6,39$ ou ( $\mathrm{N}$ total - ANP) $\times 6,39$, les résultats expriment la teneur en matière azotée totale ou la teneur en protéines du lait. En France à partir du $1^{\text {er }}$ avril 1975, la valeur azotée des laits devra, en principe, être exprimée d'après le taux de protéines. Par rapport à l'expression en 
" matière azotée totale » utilisée jusqu'alors (et encore employée à l'étranger pour le lait et d'une manière générale pour tous les produits alimentaires autres que le lait) l'expression en "protéines » se traduit par une diminution des résultats d'environ $1,6 \mathrm{~g} / 1$; elle représente la «vraie » valeur du lait en protéines.

\section{S u m m a r y}

The authors have reviewed the main factors affecting the NPN content of cow's milk.

The NPN content of milk generally lies in the range $25-30 \mathrm{mg}$ $\mathrm{N} / 100 \mathrm{~g}$ (équivalent to $1,6-2,1 \mathrm{~g}$ crude protein $(\mathrm{N} \times 6,39 \mathrm{per} \mathrm{kg}$ ). It represents 5 to 6 p. 100 of total milk nitrogen. However extreme values of 18 and $50 \mathrm{mg} \mathrm{N} / 100 \mathrm{~g}$ have been observed (table 1). These variations arise mainly from variations in the urea content of milk since the content in the other NPN components varies only very little (between 12 to $15 \mathrm{mg} \mathrm{N} / 100 \mathrm{~g}$ ) (table 1).

Urea content of milk is closely correlated and very similar to urea content of blood. It varies mainly with the total nitrogen intake of the cow : when the crude protein content of the diet increases by 1 point (p. 100), the NPN content of milk increases by $1,5 \mathrm{mg}$ $\mathrm{N} / 100$ g. Urea content of milk decreases when energy to nitrogen ratio of diet increases. With normal energy and protein intakes, increasing the porportion of NPN (with urea) in the total $\mathrm{N}$ intake, has only a small effect on the NPN content of milk.

The variation of NPN content of milk due to the diet, season, physiological state of the cow and between cows, is generally 10 times lower than that of the protein $\mathrm{N}$ content of milk.

The NPN content of milk has very little influence upon the measurement of the protein content of milk when the Amido Black method is used. However, it is more convenient to calibrate the method to true protein content (total $\mathrm{N}$ minus NPN).

Reçu pour publication en février 1975.

\section{Références bibliographiques}

Burton (H.) (1967). - Seasonal variations in deposit formation from whole milk on a heated surface. J. Dairy Res., 34, 137.

Devuyst (A.), Vanbelle (M.), Paquet (R.), Arnould (R.), Vervack (W.), Moreelsa (1964). - Les variations de la teneur en protéines du lait en fonction de l'alimentation, de la mise en prairie et de l'avancement de la lactation. Agricultura, 12, 205.

GoRdon (F. J.), Forbes (T. J.) (1970). - The associative effect of level of energy and protein intake in the dairy c w. J. Dairy Res., 37, 481. 
Grappin (R.), Jeunet (R.) (1973). - Influence de la composition de la matière azotée du lait sur la précision des dosages de matière azotée par la méthode au noir amido. Réunion du cercle International des chercheurs laitiers à Poligny les 15-16-17 mai 1973.

Hartland (H. A.), Coulter (S. T.), Jeriness (R.) (1955). - Natural variations of serum milk proteins as a limitation of their use in evaluating the heat treatment of milk. J. Dairy Sci., 38, 858.

Ide (Y.). Ogura (Y.), Yonemura (T.) (1966). - Observations on the levels of non protein and urea nitrogen in serum and milk of cows. Dairy Sci. Abs., 28, 8.

IWATA (H.) (1958). - Effects of urea and its derivatives on milk production and quality. XV Intern. Dairy Congr., 1 à 9, 54.

JARRIGE (R.) (1953). - Etude sur les variations de la richesse en constituants azotés des laits de vache. I. Influence de la mise à l'herbe. Ann. Zootech, 2, 33 .

KLOBASA (F.) (1969). - Die variabilität der inzelnen Stickstoffhaltigen Milchbestandteik und ihre abhängigkeit von verschiedenen Unweltfaktoren Tierzucht. Tiernährung, 46, $85 \mathrm{p}$.

Mac Dowell (AK. R.) (1972). - Seasonal variations in the total nitrogen, non protein nitrogen and urea nitrogen content of Friesian and Jersey milk J. Dairy Res., 39, 27.

Nickerson (T. A.). (1960), - Chemical composition of milk. J. Dairy Sci., 43, 598.

ORth, KaufmanN (1964). - Milchwissenschaft, 19, 164.

PesketT (G. L.) (1934). - Milk secretion in relation to blood ccmposition. The urea contents of blood and of milk serum. Bioch. J., 28, 1657-1658.

Reinart (A.), Nesbitt (J.M.) (1956). - The composition of milk in Manitoba. Int. Dairy. Congr., 1956, 1, (2), 946.

RENNER (E.), GoNC (S.) (1972). - Changes in the protein fractions of milk resulting from silage feeding. Wirstchaftdeigene Futter (1), 8.

RoBERTSON (N. H.), DixoN (A.) (1969). - Interrelationship between certain milk constituents. S. Afr. J. Dairy Technol., 1, 39.

RooK (J.A.F.) (1961). - Variations in the milk composition of the milk of the cow. Part Dairy Sci. Abst., 23, 251.

RooK (J. A. F.), LINE (1962). - The influence of the level dietary protein on the yield and chemical composition of milk. $16^{\mathrm{e}}$ Congrès Internat. de laiterie, vol. A, p. 57.

Rook (J. A. F.), Campling (R. C.) (1965). - Effect of stage and number of lactation on the yield and composition of cows milk. J. Dairy Res., 32, 45.

Shahani (K. M.), SOMmeR (H. H.) (1951). - The protein and non protein nitrogen fractions in milk. II. Their content in fresh raw milk. J. Dairy Sci., 34, 1010.

SEnFt (B.), KLobasa (F.) (1969). - The influence of various environmental factors on the non protein content of milk. Milchwissenschaft, 24, (12), 713.

Szijarto (L.), Biggs (P. A.), Irvine (R. M.) (1973). - Variability of casein, serum protein and non protein nitrogen in plant milk supplies in Ontario. J. Dairy Sci., 56, 45.

VenkatapPaiah (D.), Basu (K. P.) (1952). - Non protein nitrogenous constituents of milk. 1. Variation due to species, breed, individuality, season and stage of lactation. Indian J. Dairy Sci., 5, 95-116.

VERITE (R.), Journet (M.). - Résultats non publiés.

Vignon (B.), Laurent (F.) (1972). - Effet de la supplémentation des vaches laitières en urée sur la composition du lait. Bul. E.N.S.A.I.A., Nancy, XIV, 1, 13-22. 
Vignon (B.), LaURent (F.) (1969-70-71). — Résultats non publiés.

Vignon (B.), BRUn-Bellut (J.) (1972). — Résultats non publiés.

Vignon (B.) (1973). - Résultats non publiés.

VirTANEN (A. I.) (1967). - The production of milk on protein free rations. In Urea as a protein supplement. Edited by M. H. Briggs, Pergamon Press, $185-212$.

Waite, Castel (M. A.), Watson (J. N.), Drydale (A. D.) (1968). - Biuret and urea in concentrates for milking cows. J. Dairy Res., 35, 191.

WHeELock (J. V.), RooK (J. A. F.), Dodd (F. H.), GRIFFIN (T. K.) (1966). - The effect of varying the interval between milkings on milk secretion. J. Dairy Res., $33,161$.

Wheelock (J. V.), Rook (J. A. F.), Neave (F. K.), Dodd (F. H.) (1966). - The effect of bacterial infections of the udder on the yield and composition of cow's milk. J. Dairy Res., 33, 199. 